\title{
Zur Biologie der Eidechsenwurz (Sauromatum venosum)
}

\author{
G. LATZEL
}

\begin{abstract}
Tubers of Voodoo Lily (Sauromatum venosum) are sometimes offered on garden fairs. Since the tubers require neither soil nor water to produce flowers, the species is sold as a "miracle" plant. The inflorescences produce a strong odour. It attracts numerous flys belonging to species that usually deposit their eggs into dung. The paper includes observations on the flower biology of Sauromatum venosum.

Zusammenfassung

Die Knollen der Eidechsenwurz (Sauromatum venosum) werden zunehmend auf Gartenmessen angeboten. Die Pflanze wird als „Wunderblume“ verkauft, da die Knolle weder Erde noch Wasser benötigt, um einen Blütenstand auszutreiben. In den Kesselfallen-Blüten werden Fliegen gefangen, die ihre Eier normalerweise in Rinderdung legen. Einige Beobachtungen zur Blütenbiologie von Sauromatum werden vorgestellt.
\end{abstract}

\section{Heimat und Namensgebung}

Die Eidechsenwurz (Sauromatum venosum, Araceae) ist von den tropischen Bergländern Afrikas (Kamerun, Tanzania, Äthiopien und die zu Guinea gehörende Insel Fernando Po) bis nach Myanmar (Birma) verbreitet. Nach Europa gelangte sie 1848 in die Königlichen Gärten von Kew. Ihr Name bezieht sich wohl auf den gefleckten Blattstiel. „Sauros“ ist das griechische Wort für Eidechse und „chromaton“ bedeutet gefärbt wie; verkürzt wurde daraus der Gattungsname Sauromatum. Der Artbeiname venosum ist mit „venenartig gezeichnet“ zu übersetzen, was sich auf die Musterung des Blattstiels und des gefleckten Hochblattes bezieht. Eine Umbenennung der Eidechsenwurz in Typhonium venosum wird diskutiert. Die Bezeichnung Typhonium dürfte auf den schlangenleibigen, mit 100 Schlangenköpfen versehenen Riesen TyPHON zurückgehen, den die Erdgöttin Gaia dem Tartarus aus Zorn über die Vernichtung der Giganten durch ZEUs gebar; TYPHON ist laut griechischer Mythologie auch der Vater des Höllenhundes Kerberus und anderer Ungeheuer, wurde von JUPITER mit Blitzen erschlagen und liegt unter dem Ätna begraben, wo er angeblich immer wieder seinen Atem ausstößt. Noch unveröffentlichte DNA-Analysen wie auch bestimmte morphologische Charakteristika sprechen aber eher gegen eine Umbenennung (Bogner, mündl. Mitteilung).

\section{Was macht die Eidechsenwurz so interes- sant?}

Seit etwa fünf Jahren verfolgt der Autor das Angebot der Eidechsenwurz-Knollen auf der Gartenbaumesse anlässlich der Internationalen Handwerksmesse in München (IHM) und hat sie als interessantes Unterrichtsobjekt zur Demonstration des besonderen Bestäubungsmechanismus von Kesselfallen bei Aronstabgewächsen vorgestellt (s. u., LATZEL 2004, 2006).

Die Knollen der Eidechsenwurz werden unter verschiedenen Phantasienamen (z. B. Mexikanischer Wunderbaum, Wunderlilie) auf Gartenmessen mit steigenden Stückzahlen angeboten. Beim Kauf werden meist Handzettel beigelegt. Die Eidechsenwurz hat aber nichts mit Mexiko, einem Baum oder einer Lilie zu tun. Die „Blüte“ ist ein Blütenstand, der von einem auffällig gefärbten Hochblatt (Spatha) eingehüllt wird. Hauptursache des zunehmenden Verkaufserfolgs ist vielleicht die überraschende Anzuchtempfehlung zur „Blüten“-Bildung: „Ich bin eine Wunderblume, denn ich blühe ohne Wasser und Erde. Stelle mich einfach auf deine Fensterbank und ich werde dich nach kurzer Zeit (ca. 5 Wochen) mit einer wunderschönen, etwa $50 \mathrm{~cm}$ hohen Blüte beglücken."

Nach dem Verblühen muss die Knolle eingepflanzt, gegossen und nach dem Austreiben ab und zu gedüngt werden. Hat eine Befruchtung stattgefunden, entwickelt sich nach Ausbildung des Laubblattes auch der Fruchtstand mit seinen zahlreichen braunschwarzen, eckigen Beeren. 
Bis zum Herbst hat sich auf der alten Knolle eine Ersatzknolle gebildet. Reste der alten Knolle hängen noch als häutiges Gebilde an der neuen. Am oberen Rand der Ersatzknollen wachsen zusätzlich Brutknollen, die sich daraus entwickelnden Pflanzen blühen nach ein paar Vegetationsperioden. In Gebieten mit schwachen Frostperioden können die Knollen offensichtlich auch in unseren Breiten im Freien überwintern. Um zu verhindern, dass diese im Boden erfrieren, verkürzen sich die an den Knollen ansitzenden Wurzeln und ziehen die Knollen tiefer in die Erde (Bogner, mündl. Mitteilung).

\section{Blütenbiologie}

Wenn im Vorjahr ausreichend Vorratsstoffe angesammelt worden sind, können sich aus der Knolle Blütenstände entwickeln. Die Eidechsenwurz blüht im Frühjahr, erst einige Wochen später treibt das einzige grüne, bis $80 \times 80 \mathrm{~cm}$ große, fußförmig gefiederte, grüne Laubblatt aus.

Viele Pflanzenarten fördern Fremdbestäubung, indem raffinierte Mechanismen entwickelt worden sind. Eine besonders spannende Blütenbiologie zeigt die Eidechsenwurz, ähnlich wie unser heimischer Aronstab (Arum maculatum). Die Bestäuber werden in eine bauchige Falle gelockt, ohne ihnen Gegenleistungen zu bieten; die bestäubenden Fliegen werden so lange im Kessel festgehalten, bis die männlichen Blüten ihren Pollen über die Gefangenen ausgestäubt haben (MeEuse \& MORRIs 1984).

Zur Sicherung ihrer Fremdbestäubung nützt die Eidechsenwurz den Fortpflanzungstrieb von Fliegen (Eiablage auf Dung) aus, indem die Blütenstände einen intensiven Gestank nach Rinderdung aussenden, der offensichtlich für zahlreiche Fliegenweibchen (meist etwas kleiner

Abb. 1 (oben): Austreibende Knolle (im Februar/März).

Abb. 2 (Mitte): Zwei heranwachsende Blütenstände.

Abb. 3 (unten): Längsschnitt durch den Blütenstand am Morgen nach dem Aufblühtag. Der Blütenstaub ist bereits ausgeschüttet. 1: weibliche Blüten, 2: sterile, Duft produzierende Blüten, 3: männliche Blüten.
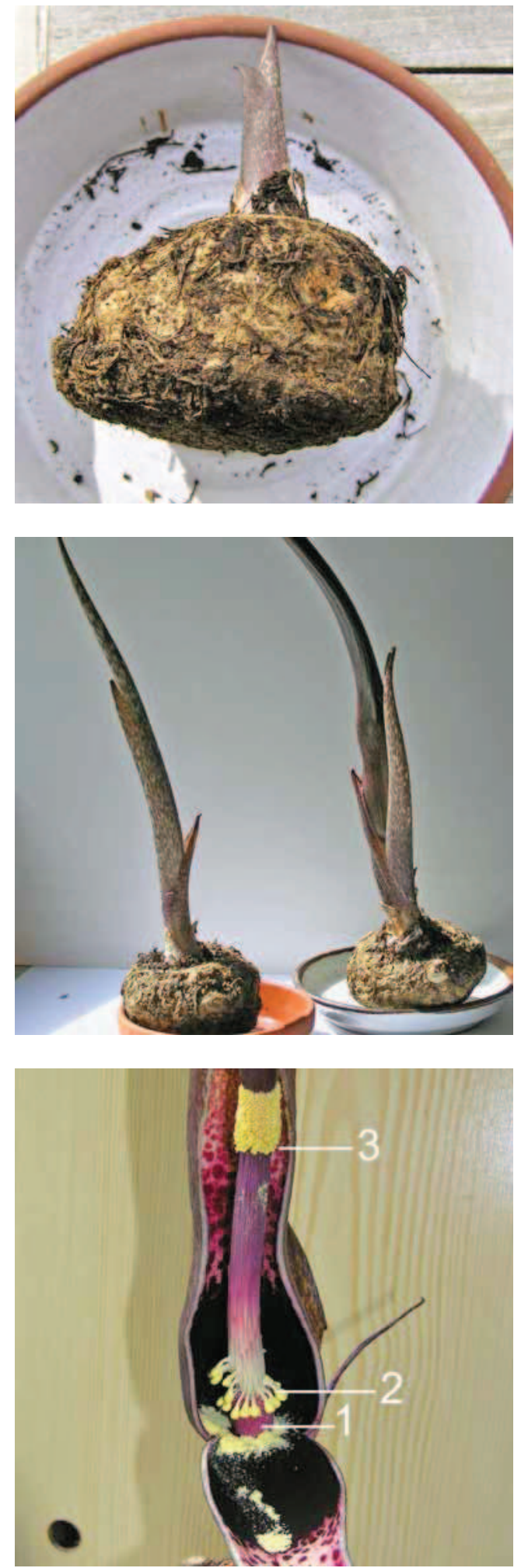

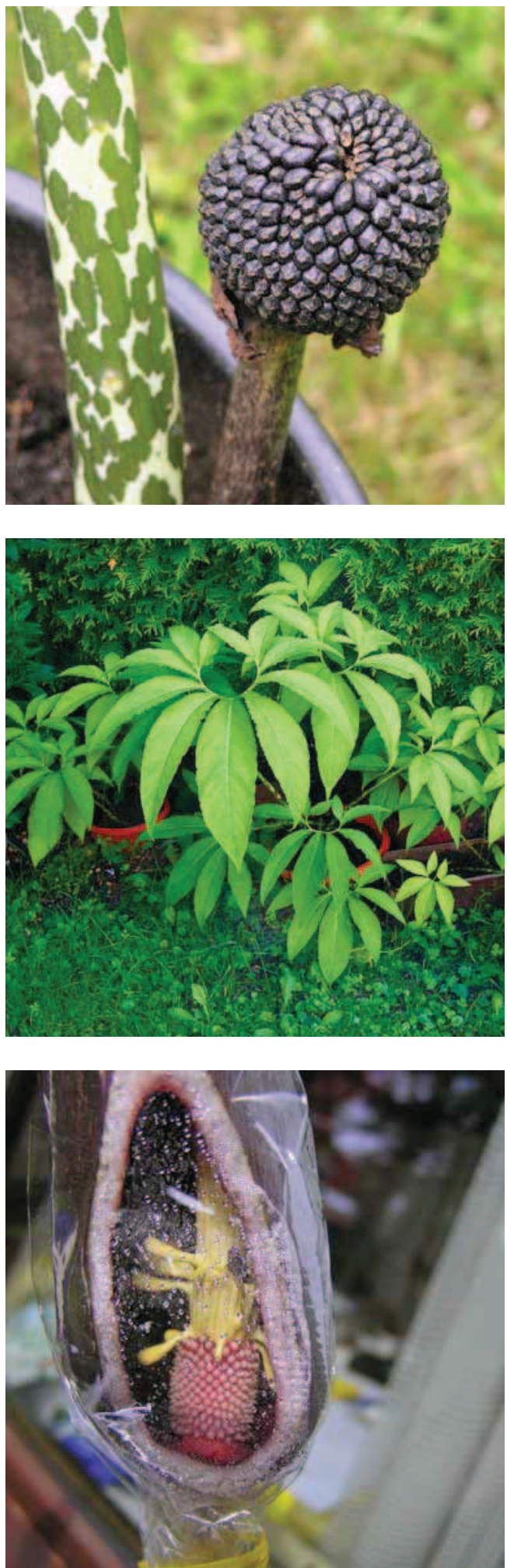

als unsere Stubenfliege) anziehend ist. Mit zwei angeschnittenen und durch eine durchsichtige Plastikfolie wieder verschlossenen Blütenständen wurden an zwei aufeinander folgenden Tagen 72 Fliegen im Freien gefangen, die alle als Weibchen vor allem verschiedener MuscidaeGattungen (67), Fanniidae (2) und Sphaeroceridae (2) bestimmt wurden. Diese Fliegen legen ihre Eier normalerweise in Rinderdung ab. Nur eine Tachinidae-Fliege bildete eine Ausnahme und könnte eventuell ein Irrläufer gewesen sein. Dieser Befund korreliert ausgezeichnet mit dem Haupt-Geruchsauslöser der Eidechsenwurz. Es handelt sich um $m$-Kresol, das einen urin-, dung-, mist- und stallartigen Geruch wie Rinderdung aufweist. Bei Temperaturzunahme wird das Verflüchtigen der Kresole deutlich verstärkt, $m$-Kresol ist überdies das einzige Kresolisomer, das bereits bei Raumtemperatur flüssig vorliegt.

Um das Verströmen des Blütenduftes (für uns eher ein Gestank) zu optimieren, kommt es zur Aufheizung des Kolbens auf Temperaturen von über $30^{\circ} \mathrm{C}$. Dies fördert die Fernanlockung der Bestäuber. Die angelockten Fliegen landen häufig auf dem „duftenden“ Anhang (Appendix) des Kolbens. Sie krabbeln dann auf der auffällig braunrot und gelb gefleckten Spatha (Dungimitation?) herum, bis sie an der oberen Öffnung der bauchigen Falle auf den Wachsschüppchen der Spatha den Halt verlieren und blitzschnell in die dunkle Falle hineinrutschen.

Abb. 4 (oben): Reifer Fruchtstand neben dem gefleckten unteren Stängelteil des Laubblattes.

Abb. 5 (Mitte): Laubblätter im Frühsommer. Im Gegensatz zu vielen anderen Aronstabgewächsen treten niemals Blütenstände zusammen mit grünen Laubblättern auf, wie das auch bei Amorphophallus der Fall ist.

Abb. 6 (unten): Der am frühen Morgen geöffnete Blütenstand ermöglicht die künstliche Fremdbestäubung. Nach dem Verschluss mit Plastikfolie kann man die weiteren Geschehnisse in der Falle verfolgen. Man sieht bereits tagsüber gefangene Fliegen auf den weiblichen Blüten und bei den keulenförmigen sterilen Blüten, die einen frischen, zitrusartigen Duft ausströmen. Als hauptsächliche Geruchsstoffe wurden Linalool (Citrus) und $\alpha$-Phellandren (Dill) identifiziert. 
Falls die Fliegen von einem vorhergehenden Blütenbesuch passenden Blütenstaub mitbringen, hinterlassen sie die Pollenkörner auf den nur wenige Stunden empfängnisbereiten Narben der weiblichen Blüten. Dass keine Selbstbestäubung stattfindet, konnte der Autor im Experiment nachweisen. Trotz der reichen Pollenausschüttung wurden nur in denjenigen Blütenständen Früchte ausgebildet, die zur empfängnisbereiten Zeit künstlich mit Pollen der ersten aufgeblühten Pflanze mit einem Pinselchen bestäubt worden waren. Der Pollen behält bei etwa $8{ }^{\circ} \mathrm{C}$ im Kühlschrank seine Keimfähigkeit bei.

Die Fliegen müssen nun bis zu 16 Stunden in der Kesselfalle zubringen, bis die männlichen Blüten ihren Blütenstaub am nächsten Morgen auf sie haben herabrieseln lassen. Damit die Fliegen in der Nacht nicht entkommen, legt sich am Abend die Spatha eng um den Kolben. Der Autor konnte im aufgeschnittenen Innenraum des Blütenstandes ca. $25^{\circ} \mathrm{C}$ messen bei knapp $16^{\circ} \mathrm{C}$ Umgebungstemperatur. Die Gefangenen bleiben warm, so dass sie am nächsten Tag sofort beweglich sind, wenn die Falle kurz nach Tagesanbruch durch den herabsinkenden Appendix wieder geöffnet wird. Das Absinken des Appendix scheint mit dem Verbrauch von Vorratsstoffen im Kolben im Bereich des männlichen Blütenstandes zusammenzuhängen, wobei zwar Erwärmung, aber keine Geruchsausströmung erfolgt. Gleichzeitig wird offenbar auch die Oberfläche der Spatha wieder begehbar.

Bei Sauromatum venosum fehlen im Gegensatz zu den bekannteren Arum-Arten (A. maculatum, $A$. italicum) die Hindernisborsten über den männlichen Blütenständen. Deren Aufgaben werden durch das Schließen bzw. Öffnen der Spatha übernommen.

Abb. 7 (oben): Fliegen auf der gefleckten Spatha (Dungimitation).

Abb. 8 (unten): Geschlossener Blütenstand am Abend des Blühtages. Die Spatha rollt sich eng um Kolben und Appendix.
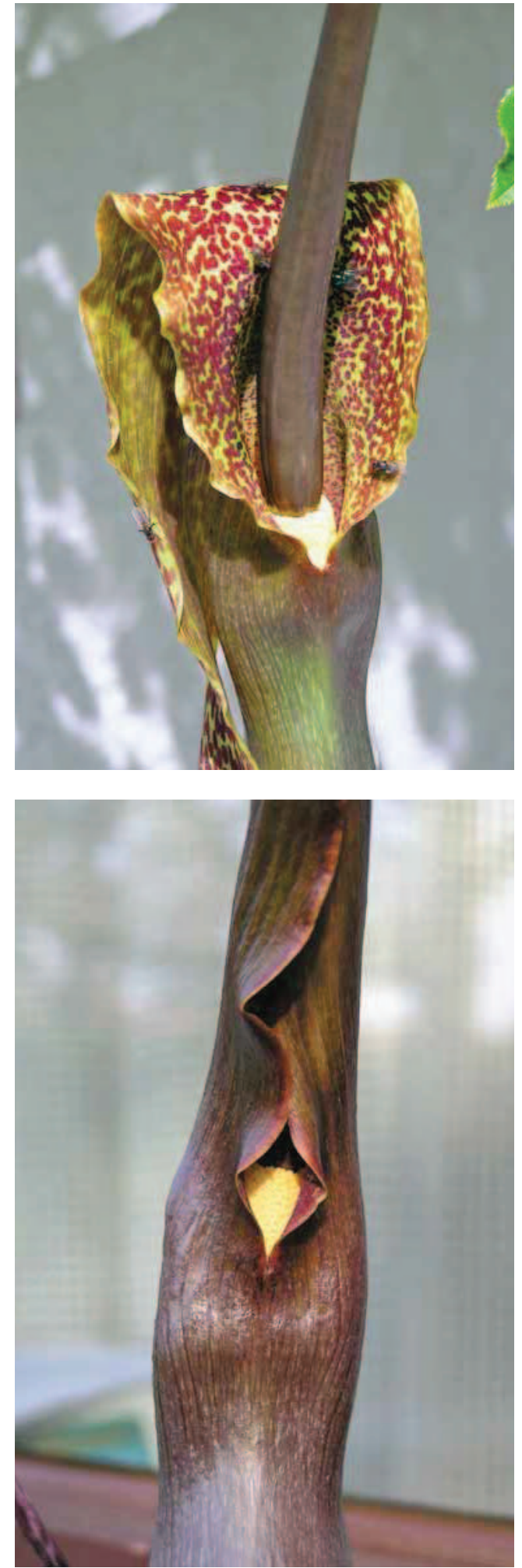

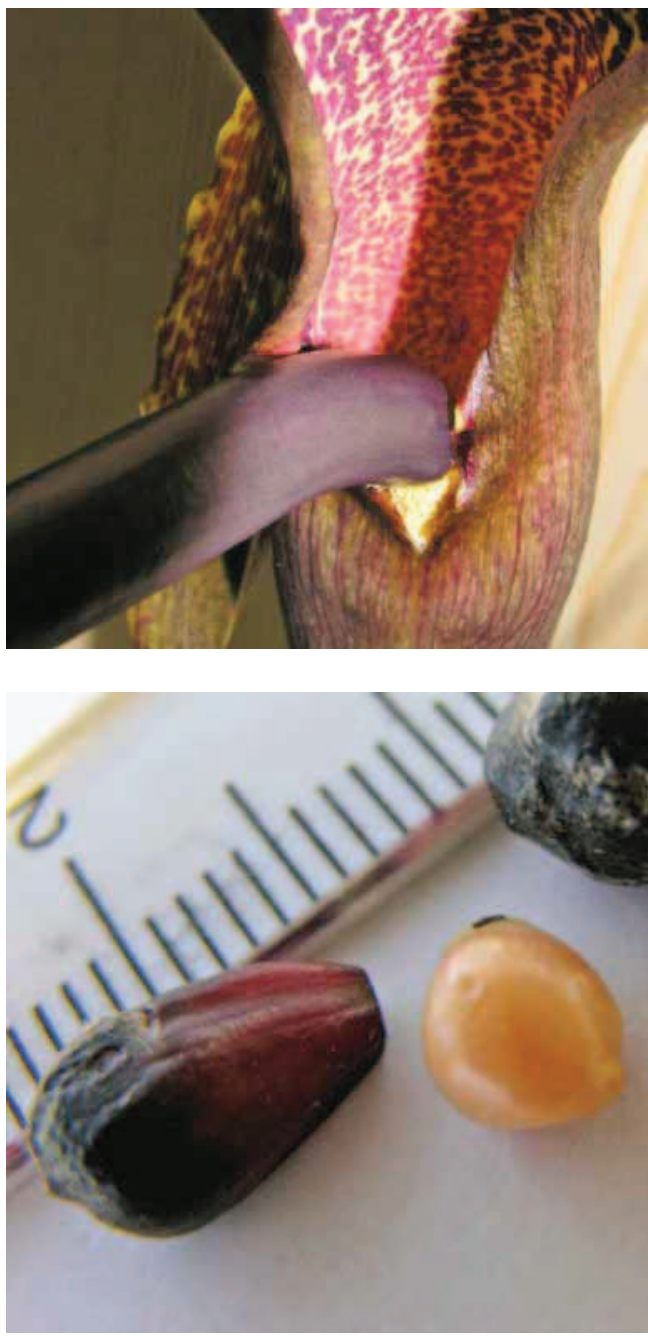

Dass offensichtlich ausschließlich Fliegenweibchen von der Eidechsenwurz angelockt werden, ist nach den Geruchsstoffen nicht überraschend. Schließlich ist Rinderdung das Substrat für die Eiablage der oben beschriebenen Fliegenarten. In den Blütenständen der Eidechsenwurz werden erstaunlicherweise jedoch keine
Eipakete gefunden, wie von anderen Araceen beschrieben (MeEuse \& MorRIs 1984). Wurden sie bisher nur übersehen oder wird die Eiablage aktiv verhindert? Zumindest gäbe es einen plausiblen Grund, warum Eipakete bei der Eidechsenwurz nachteilig sein könnten. Die bestäubten Blütenstände ruhen mehrere Monate, bis es zur Fruchtbildung kommt und Eipakete könnten im feucht-warmen, tropischen Klima von Bakterien oder Pilzen befallen werden. Die ausbleibende Eiablage könnte möglicherweise auch darauf zurückzuführen sein, dass der Aufenthalt der Fliegen relativ kurz ist oder die Fliegen durch den frischen, zitrusartigen Duft, der den keulenförmigen sterilen Blüten entströmt, eher zur Nahrungssuche als zur Fortpflanzung angeregt werden.

Welkende Blätter kündigen dann im Herbst das Ende der Vegetationsperiode an. Jetzt sollte man die Knollen frostsicher überwintern für eine neue Blüh- und Wachstumsphase.

\section{Dank}

Großer Dank gilt Wolfgang Schacht (Zoolog. Staatssammlung München) für die systematische Bestimmung der Fliegen. Dr. Florian Mayer und Dr. Andrea Burdack-Freitag (Fraunhofer-Institut für Bauphysik, Holzkirchen) bestimmten dankenswerterweise die Geruchsstoffe. Diesen beiden und dem AraceenSpezialisten Josef Bogner (LMU, München) verdankt der Autor viele anregende Hinweise und die kritische Durchsicht des Manuskripts.

\section{Literatur}

LATZEL, G. 2004: Der Mexicanische „Wunderbaum”ein Aronstabgewächs. - PdN-BioS 53(5): 42. Latzel, G. 2006: Der Mexicanische Wunderbaum heißt eigentlich Eidechsenwurz. - PdN-BioS 55(2):45. Meeuse, B. \& Morris, S. 1984: Blumen - Liebe, Sexualität und Entwicklung der Pflanzen. - Köln. 